\title{
The Influence of FDI from Different Sources on the Innovation Performance of China's Automobile Industry
}

\author{
Li zhao ${ }^{1, a, *}$ \\ ${ }^{1}$ School of Economics and Management of Northwestern Polytechnical University Mingde \\ College,Xi'an,Shaanxi,China \\ anwpulizhao@163.com
}

Keywords: FDI, Technology spillovers, Innovation performance.

\begin{abstract}
With the development of economic globalization and regional economic integration, foreign direct investment (FDI) has become the main approach of international capital flows and international technology diffusion, and attribute to its spillover effects, the FDI is employed as primary objective of attracting foreign invsestment for the host country to promote domestic technological progress. Since the reform and opening up, the FDI has grown in large scale with the gradual improvement of China's economic openness. With the deepening process of global integration, the world's auto giants standing in the core of global value chain begin to realize the fact of increasingly saturated domestic markets and cost pressure, and have to transfer its industrial center. China attracts a large number of FDI by its huge market potential and advantage of abundant cheap labor. Mass FDI inflows based on Multinational companies could on the one hand help ease the shortage of funds and technical bottlenecks in the development of China's automobile industry, but on the other hand introduce the competition pattern of global market which would strike on the market structure and competition pattern on China's auto industry. With the rapid expansion of the FDI, an extensive discussion concerning the impact of FDI on the technological innovation of the automobile industry was carried out academicly and politically, and an increasingly people hold attitudes towards the effect of foreign investment from initial exaggeration to currently suspicion or even deny.
\end{abstract}

In this paper, a study was carried concerning the influence of FDI from different sources on the innovation performance of China's automobile industry. The general development and innovation status of China's auto industry was firstly discussed, after which the time series data (1993-2017) of China's auto industry was engaged, and then investigations were conducted upon quantity of patent applications and measurment of new products output value. The conclusion suggests that FDI plays a role promoting the innovation performance of China's automobile industy through technology spillovers, and owing to cultural differences of home country, the FDI of various sources exhibits significant differences in technological innovation, technology transfer, the way into host market as well as its industry strategy, which is illustrated by the fact that FDI from opening culture like the Europe and the United States has an overweigh promotion effect compared to the closed culture like Japan and Korea in China's auto industry innovation.

As a developing country, China should expand the scale of foreign capital, make the best use of the FDI channel to attract advanced technology from developed countries actively and adjust the structure of attracting foreign capital continually, enlarge the proportion of the foreign investment based on technological innovation as the Europe and the US companies, encourage multinational corporations cooperate with China auto companies in the form of joint ventures, which would result in the maximize of the benefits brought by technology spillover effects and thus help China's auto companies obtain a better position and ownership in the global division of labor by means of technological innovation. 


\title{
不同来源地的FDI对我国轿车产业创新绩效的影响
}

\author{
李昭 $1, \mathrm{a}$, \\ ${ }^{1}$ 西北工业大学明德学院经济与管理学院, 西安, 陕西, 中国 \\ anwpulizhao@163.com
}

关键词: FDI; 技术外溢; 创新绩效

中文摘要. 随着经济全球化和区域经济一体化的发展, 外商直接投资成为国际资本流动和国际 间技术扩散的主要方式，利用外商直接投资的技术外溢效应促进本国技术进步成为东道国吸 引外资的首要目标。改革开放以来, 随着我国对外开放程度的逐步提高, 外商直接投资大规 模进入。随着全球一体化进程的不断加深, 处于全球价值链核心的世界汽车巨头面对日趋饱 和的国内市场和成本压力, 纷纷转移产业中心, 我国凭借丰富廉价的劳动力优势和巨大的市 场潜力, 吸引了大批外商来华直接投资。以跨国公司为载体的外商直接投资的大量流入，一 方面缓解了我国汽车产业发展过程中的资金短缺和技术瓶颈，另一方面将世界市场的竞争格 局传导到我国，对我国汽车产业市场结构和竞争格局产生深远的影响。随着外商直接投资规 模的迅速扩大，学术界和政策界关于外商直接投资对汽车产业技术创新的影响进行了广泛的 讨论，越来越多的人从最初对外资作用的某种盲目夸大到现在的怀疑甚至否定。

本文从我国汽车产业发展概况及创新现状出发, 利用中国汽车工业 1993-2017 年的时间序列 数据, 从专利申请量和新产品产值两个角度分别考察不同来源地的外商直接投资与我国汽车 产业创新绩效之间的关系。发现外商直接投资通过技术外溢对我国汽车产业创新绩效具有促 进作用，且不同来源地外商直接投资基于母国文化差异，在技术创新、技术转让、东道国市 场进入方式及产业战略上存在明显差异, 以欧美为代表的开放性文化较日韩等封闭性文化国 家的外商直接投资对我国汽车产业创新的促进作用更大。

作为一个发展中国家, 应扩大引资规模, 充分利用外商直接投资这一渠道主动吸收发达国家 先进技术, 同时不断调整引资结构, 扩大以技术原创为特征的欧美企业外资比重, 鼓励跨国 公司在与我国汽车企业合作过程中采用合资形式, 最大化技术外溢效应带来的好处, 促使我 国汽车企业通过技术创新在全球分工中获取更有利的地位和主导权。

\section{1. 引言}

汽车产业作为我国经济发展的支柱行业, 伴随着全球一体化进程的不断加深, 处于全球 价值链核心的世界汽车巨头面对日趋饱和的国内市场和成本压力, 纷纷转移产业中心, 我国 轿车业成为外商直接投资较多的行业。以跨国公司为载体的外商直接投资的大量流入，一方 面缓解了我国轿车产业发展过程中的资金短缺和技术瓶颈，另一方面将世界市场的竞争格局 传导到我国，对我国轿车产业市场结构和竞争格局产生深远的影响。

本文从外资来源地角度出发，着重考察如下几个问题：1）FDI对我国轿车产业在整体上 是否存在溢出效应, 如果存在溢出效应, 这种效应为正还是为负；2）FDI对我国轿车产业创 新绩效的外溢效应是否因为来源国（地区）的不同而存在差异。本文以外资企业母国文化特 征为基础, 分析不同来源地的FDI与我国轿车产业创新绩效之间的关系, 并提出政策建议。

\section{2. 文献综述及假说提出}

\section{1 文献综述}

20 世纪 80 年代以来，外商直接投资成为国际资本流动的主要方式，吸引外商直接投资 
(FDI) 也已成为许多发展中国家提升产业结构升级和推动技术进步的重要途径。根据国家商 务部统计, 我国实际使用外商直接投资额从1986年的 22.4 亿美元增长到2010年的1349.7 亿美 元。

随着外商直接投资规模的迅速扩大，国内外学术界关于外商直接投资对东道国经济的影 响进行了广泛的讨论。Macdougall(1960)在研究外商直接投资的一般福利效应时, 第一次明确 提出了FDI的外溢效应, 国外学术界关于外商直接投资对东道国技术创新的影响进行了大量的 理论和实证研究, 但迄今为止未达成共识。理论界普遍认为, 外商直接投资本身承载着东道 国所不具有的新技术和专有技术, 通过竞争效应、示范和模仿效应、人员培训和流动效应及 前后向关联效应(Blomstrom和Kokko,2001), 对东道国企业产生显著的技术外溢效应。 Caves(1974)通过对加拿大和澳大利亚1996年产业层面数据分析, 指出两国制造业中的劳动生 产率与行业内外资企业份额呈正相关, 即FDI对两国制造业具有正向的溢出效应。姚树洁等人 从外商直接投资影响新型工业化国家发展过程的具体机制出发，通过对1979年-2003年29个 省和直辖市面板数据的分析，指出外商直接投资在生产技术效率 “推动器” 和生产前沿 “移 动器” 的双重作用下, 对东道国经济增长产生积极影响。王成岐、张建华等通过对中国制造 业1995年和2003年的行业数据分析，指出FDI在总体上产生显著的溢出效应，但是外资企业对 内资企业绩效间的关系不是普遍而一致的, 相对于港澳台和海外华人投资, 来自西方国家外 资的溢出效应更为显著。

另外一些学者认为，外商直接投资通过 “市场窃取” 导致内资企业成本增加、生产效率 下降, 产生 “负溢出” 现象。蒋殿春和张宇将制度环境与我国目前的转型经济相结合, 指出 现有的制度缺陷弱化了国内企业学习和创新的动力, FDI技术溢出效应并不显著甚至为负。

国内外众多学者通过建立不同模型从不同层面论证了以跨国公司为载体的外商直接投资 对东道国经济发展的影响。学者们的研究基本上以FDI对东道国国内企业经济增长绩效的影响 为主, 且大多集中在产业层面, 关于FDI对东道国特定行业技术创新影响的文献则很少。目前 已有的关于FDI对东道国技术创新影响的研究大都集中在FDI对专利申请量的影响及其影响 因素分析。

\section{2 假说提出}

\subsection{1 假说一：外商直接投资对我国轿车产业创新绩效具有促进作用}

汽车产业作为我国经济发展的支柱产业, 随着经济全球化的快速发展, 处于成长期的中 国轿车业吸引了越来越多的跨国汽车生产厂商的进入。FDI的大量流入通过两个传导机制对我 国轿车产业创新绩效产生影响。一方面，外商直接投资通过技术外溢对轿车产业的技术进步 产生积极地推动作用; 另一方面, 外商直接投资的涌入通过改变轿车产业的市场集中度促进 轿车产业市场结构的改变和升级, 竞争性的市场结构迫使轿车产业加快技术进步。具体传导 机制如图:

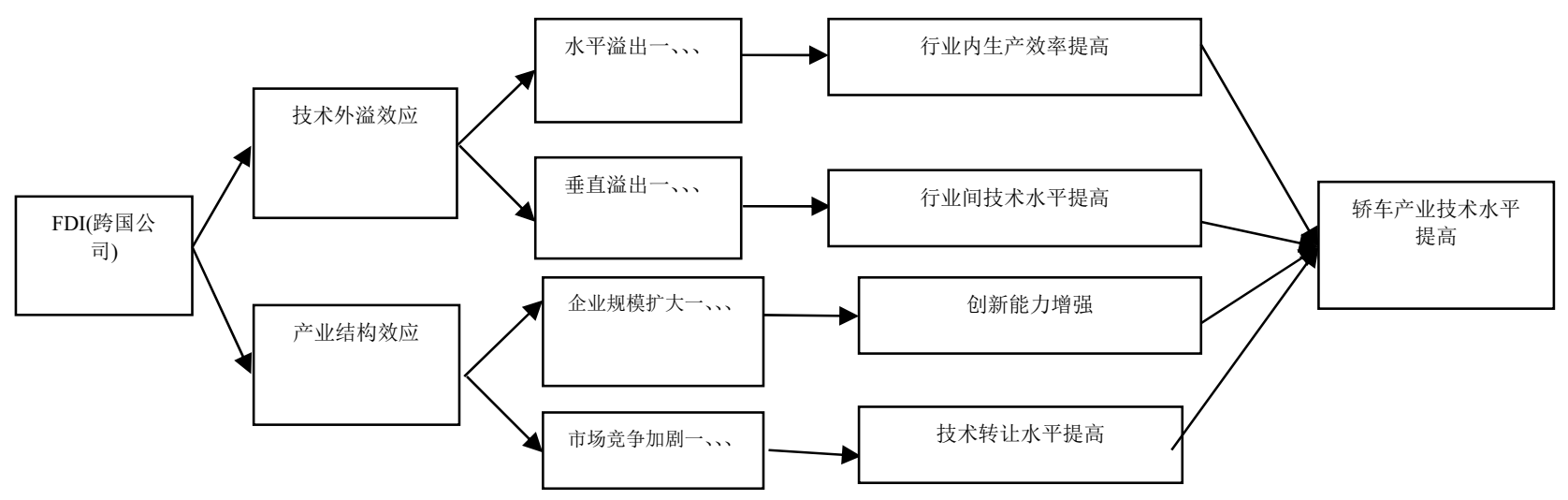

图 1 外商直接投资 FDI 对我国轿车产业创新绩效传导机制 
外商直接投资本身承载着东道国所不具有的新技术和专有技术，来华投资的绝大多数跨 国公司提供了母公司比较先进的技术，其中相当一部分填补了国内空白的技术。外资企业提 供的先进技术通过行业内的水平溢出和行业间的垂直溢出对我国轿车企业产生显著的技术外 溢效应。水平溢出主要通过竞争、示范和模仿及人员培训和流动而发生; 垂直溢出效应主要 通过外资企业与东道国上下游企业间的前后向关联而发生。东道国企业在 FDI 溢出效应发生 的不同机制下通过模仿学习、引进技术、技术援助或技术合作等技术进步路径提高技术水平。

跨国汽车生产寡头以直接投资方式进入我国汽车行业,不仅带来了汽车产业所需要的资 本和技术，同时将世界汽车产业市场的国际竞争格局传导到了中国，使我国整车产业市场结 构和竞争格局发生了显著的变化。随着外商直接投资的进入, 中国汽车产业市场已从竞争型 转向了中下集中寡占型。根据熊彼特的观点, 高集中市场中的大企业有利于创新。对于资本 密集型的汽车产业而言, 市场结构的变化对创新绩效的正效应来源于大规模生产的规模经济 性、先进的技术和设备、完善的企业组织和管理及必要的研发投入等因素。基于上述理论, 我们得到第一个假说：外商直接投资对我国轿车产业创新绩效具有促进作用

\subsection{2 假说二：相比日韩，欧美对我国轿车产业创新绩效的促进作用更强}

现有的研究通常将不同国别的外商直接投资作为一个整体来对待, 忽视了不同来源地国 家历史文化、投资动机及产业发展战略等方面可能存在很大差, 这导致不同来源地的外商直 接投资行为之间存在很大差异, 进而对东道国企业创新绩效产生不同的影响。1983年北京吉 普汽车有限公司的成立拉开了我国汽车行业外商投资的序幕,随后我国汽车工业实际使用外 资额逐年上升，其中港澳台、韩国、日本、英国、德国、美国等国家是我国外商直接投资的 主要来源地。本文根据外资企业来源地文化的不同, 将它们分为两类, 一类是以欧美为为代 表的开放性文化国家, 其对外表现出较强的包容性和互助性; 另一类是以日韩为代表的封闭 性文化国家, 对内表现出高度的民族凝聚力, 对外则表现出强烈的集团主义色彩, 但这种集 团主义强调界限清晰的小范围主义。外商直接投资作为在异质文化主体之间进行的经济活动, 母国文化特性对跨国公司行为有着根深蒂固的影响，本文从不同文化主导下的外资企业在技 术创新、技术转让、东道国市场进入方式、贯彻国家对外政策等方面考察不同来源地的FDI 对我国轿车产业创新绩效影响。

(1) 不同文化类型对技术创新的影响

以欧美为代表的开放性文化推崇个人主义，强调独立个性。个人主义和冒险主义盛行的 开放性文化激励企业家才能和创新, Ghemawat(2003)发现重大突破性技术创新较多在不严格 规避风险的国家出现。以日韩为代表的封闭性文化强调团队的协调性, 通过企业内部活动建 立企业网络提升工作效率、获得协调优势。在汽车产业的发展过程中, 日韩起步较晚, 它们 奉行以引进为基础的学习型和追赶型战略。日韩轿车企业通过引进欧美先进技术, 并对生产组 织和管理进行改革和创新, 发挥协调性优势, 推出以丰田为代表的 “精益” 生产模式, 从而在 国际汽车市场上占据了一席之地。

(2) 不同文化主导下的外资企业技术转让方式的差异

以日韩为代表的封闭性文化国家在进行对外直接投资时,基于文化的保守性对技术转让 持特别谨慎的态度。2007年3月, 日本经济产业省出台了《防止技术流出指针》, 要求日本企 业确立严格的技术管理体制, 有关企业必须把重要产品和技术用 “黑匣子” 保护起来、尖端 技术转让只限于全资子公司范围内，不得转让给合资公司，严防先进技术流失海外。罗鹏、 钱永坤通过对日在华企业日方管理人员的问卷调查发现, 日本在华直接投资企业在追求利润 的趋势下, 为了延长技术生命周期, 转让的技术总体质量不高, 技术含量低, 往往都属于一般 的或标准化的技术，甚至是陈旧过时的技术。

欧美轿车生产企业作为技术原创者, 一直利用技术的进步和垄断作为对外资本扩张中与 对手竞争、保持和扩大市场份额以及获取巨额利润的主要手段。美国跨国公司始终把技术领 先作为其发展海外经营的基础, 在华的投资企业技术水平一般比较高, 欧美跨国公司在对外 
投资中不仅显示出较强的技术优势，同时对于技术流出的限制相对于日韩企业较少。

（3）不同文化背景的外资企业与东道国合作方式的不同

开放性文化与封闭性文化背景下的外商直接投资企业与我国轿车企业合作方式截然不 同，但大都可以找到其文化渊源。封闭性文化下母国的高权力距离偏向获得控股权并且较少 在东道国R\&D（关涛、任胜刚，2009），对外投资时通常倾向于采用独资形式，这种合作形 式有效的保持了母公司的技术秘密与技术优势, 却不利于东道国技术外溢, 极大减少了我国 轿车企业通过合资获取技术知识的机会和途径。欧美等跨国公司在冒险精神和追求垄断利润 的驱使下，较早的进入我国轿车产业，投资者在东道国经营风险的不确定性使得大多数企业 倾向于选择合资形式，而开拓型合资企业对我国轿车产业的创新绩效要好于独资型企业。

(4) 不同文化主导下的国家政策的差异

从某个时点看，国家政策本身就是文化作用的产物。封闭性文化主导下的日韩实施单向 国际化策略，国内市场相对封锁；而以开放性文化为导向的欧美国家倡导双向国际化策略。 从外商投资来源地国家产业发展战略来看，不同的战略动机决定了封闭性文化导向和开放性 文化导向下的外资企业存在本质差异，进而对东道国企业的创新绩效产生实质性的影响。日 本在 20 世纪 80 年代初制定的 “雁阵发展模式” ，将自己确定为亚洲经济发展的领头雁，中国 处于 “雁行模式” 的最底层，在对外投资过程中，奉行小岛清的 “边际产业扩张理论” ，将 国内比较劣势产业转移到按 “雁阵” 顺序进行对外转移, 从而保留本国比较优势产业扩大出 口，实现 “贸易立国”。20世纪90年代后半期, 随着日本及中国经济形式的变化, 日本积极 调整产业发展战略, 加大对中国的直接投资和技术合作, 并开始在中国设立研发中心。在这 一过程中, 日本启动知识产权战略, 研发大多严防技术外流, 在海外研发也大多以适用型和 专利技术为主, 创新型研究很少。

美国的轿车产业战略以海默和金德尔伯格的垄断优势理论为基础，跨国公司在垄断利润 的驱使下，竞争对手较少的东道国比在母国市场中更容易形成垄断行为。在对外投资过程中 多用结构型创新、垄断型创新在高新技术和垄断产业开始, 投资形式也多以垄断性大企业为 主，这相对于日本 “精益生产” 生产方式下的 “低成本” 的流水线制造对我国轿车产业的创 新影响更强。基于上述分析，不难发现，以欧美为代表的开放性文化较日韩等封闭性文化国 家的外商直接投资对我国轿车产业创新的促进作用更大，因此，本文提出第二个假说：相比 日韩，欧美对我国轿车产业创新绩效的促进作用更强。 


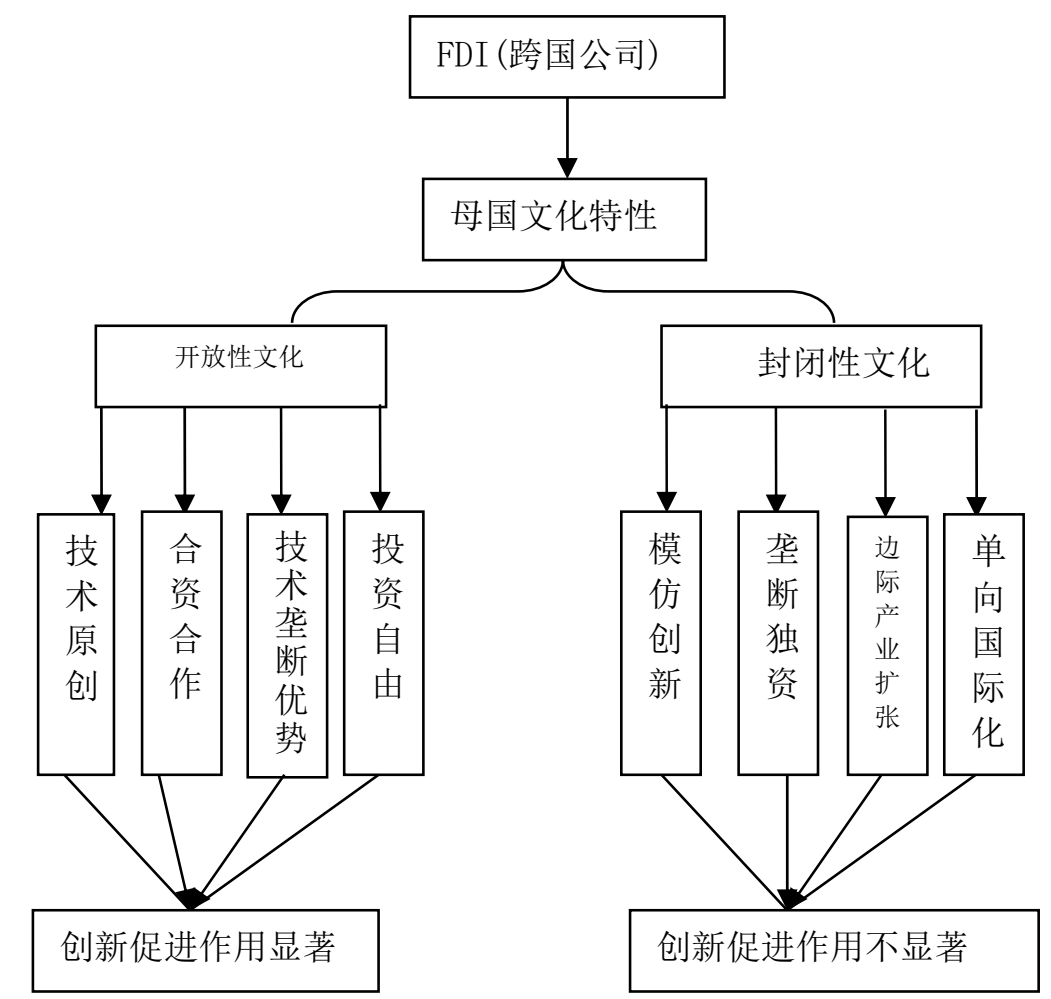

图 2 不同文化类型下外商直接投资对我国轿车产业创新绩效的影响

\section{3. 计量分析及解释}

\section{1 变量选择}

被解释变量: 本文以我国轿车产业创新绩效为被解释对象。在已有文献中，许多学者采 用专利申请量作为创新绩效的衡量指标, 专利的不完全性和同质性假设导致该项指标不能全 面反映创新成果中新产品的开发情况。在考虑产品创新的市场价值的情况下，本文同时选取 新产品销售收入比作为专利的补充, 克服用专利数量衡量创新绩效时的缺陷。所以本文的被 解释变量包括: (1) 专利申请量, 包括发明专利、实用新型以及外观设计三种专利申请量, 记为PA;（2）新产品产值, 以新产品销售收入与企业产品销售收入的比例来表示, 记为NP。

解释变量: 本文以不同来源地的 FDI 为解释变量, 记为 FDIi。为了便于分析, 本文将我 国引进外商直接投资最多的国家或地区分为四类：对华直接投资数额较大但大多以劳动密集 型的小型企业为主的港澳台地区作为第一个外资来源地, 记为 FDI1; 日本、韩国的汽车产业 起步较晚, 它们通过引进西方国家先进技术不断模仿创新而成功的国家, 将它们作为第二个 外资来源地, 记为 FDI2; 以美国、加拿大为代表的来源于北美地区的外商直接投资作为第三 个外资来源地, 记为 FDI3; 英国、德国与法国三个欧洲国家作为第四个外资来源地,记为 FDI4。

为了准确分离出不同来源地的 FDI 对我国汽车产业创新绩效的影响, 还需要考虑其他可能 影响创新的因素。这些主要因素有：1）企业规模：按照熊彼特的创新假设，大企业因具备较 高的承担风险的能力, “已成为那种进步的最强有力的机器”。对于规模经济显著的汽车行业 而言, 企业规模是影响创新绩效的重要因素。由于销售收入在生产要素构成中处于中立地位, 同时又是企业研发的根据。因此，本文以企业销售收入作为企业规模的衡量指标，记为 SIZE。 2) $\mathrm{R} \& \mathrm{D}$ 投入: 必要的资金和人员投入是进行科研活动的基础, 也是提高研发水平的重要手 段, 本文将 R\&D 投入作为影响创新绩效的重要指标, 记为 RD；3）市场结构: 本文选取市 场集中度 $\mathrm{CRn}$ 作为刻画产业市场结构的指标，同时结合目前我国汽车业中一汽、上汽、东风、 广汽四家厂商占据较大市场份额的现状, 以 CR4 衡量市场垄断程度和企业市场控制力, 记为 $\mathrm{CR}$ ；4)产权结构: 我国目前正处于经济转型期, 市场经济尚不成熟, 制度因素对创新行为影 
响深刻。本文分别从国有企业和非国有企业角度考察产权结构, 以国有企业与非国有企业产 值之比重表示产权变量, 分别记为 $\mathrm{OWN}, \xi$ 为残差项。

表 1 主要变量的定义和符号

\begin{tabular}{|c|c|c|c|}
\hline \multicolumn{2}{|c|}{ 变量 } & 定义 & 符号 \\
\hline \multirow{2}{*}{ 被解释变量 } & 专利 & 专利申请量 & PA \\
\hline & 新产品产值 & 新产品销售收入/企业产品销售收入 & NP \\
\hline \multirow{5}{*}{ 解释变量 } & \multirow{5}{*}{ 外商直接投资 } & 实际利用外商投资额 & FDI \\
\hline & & 来自港澳台外商直接投资额 & FDI1 \\
\hline & & 来自日韩的外商直接投资额 & FDI2 \\
\hline & & 来自美国、加拿大的外商直接投资额 & FDI3 \\
\hline & & 来自英、德、法的外商直接投资额 & FDI4 \\
\hline \multirow{4}{*}{ 控制变量 } & 企业规模 & 企业销售收入/企业个数 & SIZE \\
\hline & $\mathrm{R} \& \mathrm{D}$ 投入 & 企业研发活动实际投入资金总额 & $\mathrm{RD}$ \\
\hline & 产权结构 & 国有企业产值/非国有企业产值 & OWN \\
\hline & 市场结构 & 市场集中度CR4 & $\mathrm{CR}$ \\
\hline
\end{tabular}

为了便于分析, 分别对上述指标取对数。我们构造如下模型来分析不同来源地的外资对 我国轿车产业创新绩效的影响:

$$
\begin{aligned}
& \log \mathrm{PA}=\beta_{0}+\beta_{1} \log \mathrm{FDI}_{i}+\beta_{2} \operatorname{logSIZE}+\beta_{3} \log \mathrm{RD}_{i}+\beta_{4} C R+\beta_{5} \log \mathrm{OWN}_{\mathrm{i}}+\xi \\
& \log \mathrm{NP}=\beta_{0}+\beta_{1} \log \mathrm{FDI}_{i}+\beta_{2} \operatorname{logSIZE}+\beta_{3} \log \mathrm{RD}_{i}+\beta_{4} \mathrm{CR}+\beta_{5} \log \mathrm{OWN}_{\mathrm{i}}+\xi
\end{aligned}
$$

\section{2 描述性统计分析}

表 2 主要变量的统计性描述

\begin{tabular}{ccccccccc}
\hline 变量名称 & 单位 & 观察值 & 均值 & 中位数 & 最大值 & 最小值 & 标准差 & 相关系数 \\
\hline PA & 个 & 18 & 4812 & 4000.5 & 15646 & 1522 & 3977.1 & 1 \\
$\mathrm{NP}$ & $\%$ & 18 & 0.35 & 0.37 & 0.45 & 0.23 & 0.07 & $(1)$ \\
$\mathrm{FDI}$ & 亿美元 & 18 & 15.3 & 11.6 & 34.1 & 3.1 & 10.3 & $0.61(0.71)$ \\
FDI1 & 万美元 & 18 & 48329.1 & 39627.1 & 160676.5 & 14799.5 & 33508.3 & $0.77(0.31)$ \\
FDI2 & 万美元 & 18 & 32314.3 & 14520.9 & 128886.8 & 2855.5 & 36436.2 & $0.31(0.58)$ \\
FDI3 & 万美元 & 18 & 13738.1 & 10116.4 & 38534.4 & 3224.1 & 10254.1 & $0.13(0.51)$ \\
FDI4 & 万美元 & 18 & 13267.9 & 5577.5 & 59728.1 & 785.1 & 15840.7 & $0.37(0.55)$ \\
SIZE & 万元 & 18 & 491146.2 & 301105.3 & 1701586 & 119385.1 & 459842.1 & $0.89(0.71)$ \\
RD & 万元 & 18 & 835652.6 & 450561 & 2705666 & 76868 & 865995.3 & $0.82(0.72)$ \\
OWN & $\%$ & 18 & 0.76 & 0.46 & 2.35 & 0.12 & 0.68 & $-0.56(-0.86)$ \\
CR4 & $\%$ & 18 & 49.2 & 47.1 & 58.5 & 39.2 & 7.3 & $0.52(0.37)$ \\
\hline
\end{tabular}

注: 相关系数指标的描述性统计中括号内为各解释变量、控制变量与被解释变量NP之间的相关关系, 括号 外为各变量与被解释变量PA之间的相关关系。

从不同来源地外商直接投资额的均值和中位数来看，来自日韩的投资额在各年之间波动 明显大于其它来源地的外商直接投资额，间接支持了前文的推断一一基于封闭性文化背景下 的日韩企业在做投资时非常谨慎, 中日关系的变动对日本企业的投资动向有较大影响。企业 
$\mathrm{R} \& \mathrm{D}$ 投入、产权结构等指标均值与中位数之间、最大值与最小值之间的差异较大，表明企业 在R\&D投入、产权结构等方面存在较大的差异。

通过观察相关系数，我们发现外商投资、企业规模、 $R \& D$ 投入与新产品产值与专利申请 量之间存在显著地正相关关系, 初步肯定了命题一; 产权结构变量与创新绩效之间呈负相关 关系, 我们认为这种现象是源于国有企业对创新的促进作用较小。

\section{3 模型设定及检验}

\subsection{1 模型设定}

根据前文的研究，企业规模和R\&D投入与企业创新绩效具有显著地相关性，同时我们结 合目前我国汽车产业现状, 引入产权结构与市场结构变量考察外商直接投资对我国汽车产业 创新绩效的影响。因此，我们构造如下模型来验假说一：

$$
\begin{aligned}
& \ln P A=\beta_{1} \ln F D I+\beta_{2} \ln R D+\beta_{3} \ln S I Z E+\beta_{4} \ln O W N+\beta_{5} \ln C R 4 \\
& \ln N \mathrm{P}=\beta_{1} \ln F D I+\beta_{2} \ln R D+\beta_{3} \ln S I Z E+\beta_{4} \ln O W N+\beta_{5} \ln C R 4
\end{aligned}
$$

同时，为了进一步考察不同来源地外商投资对汽车产业创新绩效的影响，我们构造如下 模型验证假说二:

$$
\begin{aligned}
& \ln P A=\beta_{1} \ln F D I_{1}+\beta_{2} \ln F D I_{2}+\beta_{3} \ln F D I_{3}+\beta_{4} \ln F D I_{4} \\
& \ln N P=\beta_{1} \ln F D I_{1}+\beta_{2} \ln F D I_{2}+\beta_{3} \ln F D I_{3}+\beta_{4} \ln F D I_{4}
\end{aligned}
$$

\subsection{2 检验及结果分析}

单位根检验：本文采用时间序列进行分析，为了提高检验功效、防止对非平稳变量进行

\begin{tabular}{|c|c|c|c|c|c|c|c|}
\hline 变量 & 差分次数 & $(\mathrm{C}, \mathrm{T}, \mathrm{K})$ & DW 值 & ADF 值 & 5\%临界值 & 1\%临界值 & 结论 \\
\hline $\ln \mathrm{PA}$ & 1 & $\left(\begin{array}{lll}0, & 0, & 0\end{array}\right)$ & 2.053 & -5.794 & -1.964 & -2.718 & I (1) \\
\hline $\operatorname{lnNP}$ & 1 & $(0,0,1)$ & 1.801 & -2.473 & -1.966 & -2.728 & I (1) \\
\hline $\operatorname{lnFDI}$ & 1 & $(0,0,2)$ & 1.975 & -2.012 & -1.968 & -2.741 & I (1) \\
\hline $\operatorname{lnFDI1}$ & 1 & $(0,0,1)$ & 1.744 & -3.093 & -1.966 & -2.728 & I (1) \\
\hline $\operatorname{lnFDI} 2$ & 1 & $\left(\begin{array}{lll}0, & 0, & 0\end{array}\right)$ & 1.603 & -2.464 & -1.964 & -2.718 & I (1) \\
\hline $\operatorname{lnFDI3}$ & 1 & $\left(\begin{array}{lll}0, & 0, & 0\end{array}\right)$ & 1.832 & -3.933 & -1.964 & -2.717 & I (1) \\
\hline $\operatorname{lnFDI} 4$ & 1 & $\left(\begin{array}{lll}0, & 0, & 0\end{array}\right)$ & 1.731 & -3.329 & -1.964 & -2.717 & I (1) \\
\hline $\ln R D$ & 1 & $(\mathrm{C}, 0,0)$ & 2.143 & -4.858 & -3.066 & -3.921 & I (1) \\
\hline $\operatorname{lnSIZE}$ & 1 & $(\mathrm{C}, 0,0)$ & 1.95 & -3.065 & -3.920 & -4.233 & I $(1$ \\
\hline $\operatorname{lnOWN}$ & 1 & $\left(\begin{array}{lll}0, & 0, & 0\end{array}\right)$ & 2.011 & -4.454 & -1.964 & -4.453 & I (1) \\
\hline $\operatorname{lnCR} 4$ & 1 & $(0,0,0)$ & 1.395 & -2.978 & -2.717 & -1.964 & I (1) \\
\hline
\end{tabular}
伪回归, 我们对各变量进行单位根检验。各变量本身非平稳, 对其进行一阶差分处理后得到 平稳序列，结果如表3所示。

表 3 单位根检验结果

协整检验：对各变量进行差分使其平稳，但使我们失去总量的长期信息，为了分析变量 之间的长期稳定关系，本文通过构造VAR模型，观察模型全部根的到数值均在单位圆内，同 时利用滞后长度准则确定最佳滞后期，在此基础上采用Johansen协整检验分析变量之间的长 期均衡关系。我们首先对假说一进行检验, 分析外商投资在整体上对我国汽车产业创新绩效 的影响。 
$\ln P A=0.1311 \ln F D I+0.5761 \ln R D+0.2971 \ln S I Z E-0.7111 \ln O W N+0.3281 \ln C R 4$

$$
\ln N \mathrm{P}=0.361 \ln F D I+0.5651 \ln R D+0.5031 \ln S I Z E-0.4741 \ln O W N+0.2191 \ln C R 4
$$

根据检验结果, $\operatorname{lnFDI}$ 的系数均为正, 说明FDI对我国汽车产业创新具有促进作用, 但对 汽车产业新产品销售收入的促进作用远大于对专利申请量的影响, 我们推测这与我国知识产 权法律法规体系及知识产权保护环境的不完善有着密切的关系。我们在模型中添加了影响创 新绩效的另外两个显著因素, 同时根据我国经济环境特征加入产权结构和市场结构变量, 发 现企业规模和R\&D投入对创新有促进作用，这种作用的发挥要以合理的企业所有制或公司治 理结构为前提。文中 $\operatorname{lnCR} 4$ 的系数为正, 意味着随着汽车产业市场集中度的逐步提高, “市场 势力”为企业创新提供内生的动力机制、盈利机制和再投入保障机制。

对假说二进行检验，考察不同来源地FDI对创新绩效之间的影响，根据协整检验的结果， 各变量之间存在如下长期关系:

$$
\begin{gathered}
\ln P A=-0.2261 \ln F D I_{1}+0.5341 \ln F D I_{2}+1.054 \ln F D I_{3}+0.6241 \ln F D I_{4} \\
\ln N P=1.468 \ln F D I_{1}+1.181 \ln F D I_{2}+2.657 \ln F D I_{3}+3.477 \ln F D I_{4}
\end{gathered}
$$

来自港澳台的外商投资与汽车产业新产品产值之间具有正相关性，与专利申请量之间却 存在负相关关系, 因为来自港澳台企业大多以劳动密集型小企业为主, 本身技术水平不高, 且大多处于汽车产业链的下游环节。1nFDI2、lnFDI3 和1nFDI4的系数均为正, 但 $1 n$ FDI3 和 $1 n$ FDI4 的系数明显较大, 说明来自美国、加拿大和英德法的外商投资对我国汽车产业创新绩效的促 进作用更强, 与前文命题二的假说一致。

\section{4. 结论及建议}

本文从专利申请量和新产品产值两个角度考察了来自不同国家的外商直接投资对汽车产 业创新绩效进行实证研究, 结果发现: 外商直接投资通过技术外溢效应和产业结构效应对我 国汽车产业创新绩效具有促进作用; 不同来源地的外商直接投资基于母国文化差异, 在技术 创新、技术转让、东道国市场进入方式及产业战略上截然不同，分析表明，以欧美为代表的 开放性文化较日韩等封闭性文化国家的外商直接投资对我国汽车产业创新的促进作用更大。

本文的研究结果具有重要的政策含义。首先：外资直接投资总体上具有显著地促进作用。 近年来学术界和政策界对汽车产业 “以市场换技术” 的实际效果有较大争议, “越来越多的人 从最初对外资作用的某种盲目夸大到现在的怀疑甚至否定 (王成岐、张建华、徐文忠, 2006)”。 部分人断言伴随着以大产业、大整合为特征的外资的涌入，我们不仅无法获得技术甚至 “丢 掉市场”。本文从专利申请量和新产品产值两个角度肯定了外商直接投资通过技术外溢效应和 产业结构效应对汽车产业创新绩效具有促进作用，对 “以市场换技术” 政策的争论提供了有 意的参考。正如王成岐等人所言, 目前的问题不在于要不要外资, 而是如何利用外资为政策 目标服务。作为一个发展中国家, 我国汽车产业起步较晚, 应以推动技术进步、加快产业结构 升级, 提高市场势力作为引进外资的目标, 充分利用直接投资这一渠道主动吸收发达国家先 进技术, 促使我国汽车企业通过技术创新在全球分工中获取更有利的地位和主导权。

其次，来自欧美国家的外资对创新的促进作用更强。从外资来源地看，以外资实际使用 额计算，港澳台和日韩一直位居汽车工业吸收外资来源地的前列。来自港澳台的外资大多以 劳动密集型的小企业为主, 对我国汽车产业技术转让有限; 以追求最佳成本收益为目标的日 韩企业在华投资的主要动机在于廉价劳动力的保证, 母国企业垄断优势核心技术, 为避免在 “家门口” 培养出能与自己抗衡的强敌, 往往将技术含量低的生产制造环节转移到中国。以 “市场为导向” 的欧美企业为了占领迅速成长的东道国市场需求空间, 将先进的技术引入, 同时组建研发中心, 提高企业创新能力和生产效率, 谋求更好的发展空间。因此, 政府在引 资过程中不仅要关注引资规模, 更要关注引资结构, 鼓励跨国公司在与我国汽车企业合作过 
程中采用合资形式，最大化外资溢出带来的好处。

\section{References}

[1] Wang cheng qi.Zhang jian hua.Xu wen zhong. Who Benefits from: Foreign Direct Investment in China's Manufacturing Industry . China Industrial Economics, vol.10, pp. 23-31, 2006

[2] Bo guang wen. The Impact of FDI on China's Technological Innovation: A Regional Study . Journal of Finance and Economics, vol.6, pp. 4-17, 2016

[3] Xian guo ming.Bo guang wen . The Impact of FDI on China's Technological Innovation: An Analysis Based on the Industrial Level . Word Economy Studies, vol.6, pp. 16-22, 2015

[4] Wu yan bin. Determinants of Innovation: An Empirical Study Based on China's Manufacturing Industry. World Economic Papers, vol.2, pp. 46-57, 2008

[5] Yao shu jie.Feng gen fu.Wei kai lei. A Study on the Relationship between Foreign Direct Investment and Economic Growth.Economic Research Journal , pp. 35-47, 2016

[6] Blomstrom M, Kokko A. Foreign Direct Investment and Spillovers of Technology.International Journal of Technology Management, 2001(22): 435-454

[7] Caves R E Multinational firms ,competition and productivity in host-country markets.Economica,1974,(41):176-193

[8] Shen kun rong.Geng qiang. Foreign Direct Investment, Technology Spillovers and Endogenous Economic Growth: A Quantitative Test and Empirical Analysis of China's Data.Social Sciences In China, vol.5, pp. 82-102, 2011

[9] Jiang dian chun.Zhang yu. Economic Transition and Technology Spillover Effect of FDI . Economic Research Journal, pp. 26-39, 2008

[10] Hu A, Jefferson G. FDI, Technology innovation and Spillover:Evidence from large and medium size Chinese enterprises, [M].Brandeis University, 2001

[11]Jiang xiao juan.Multinational Investment, Market Structure and Competitive Behavior of Foreign-funded Enterprises. Economic Research Journal, vol.9, pp. 31-41, 2012

[12]China Automotive Industry Yearbook [G]. China Automobile Industry Association.1986 2018. 